\section{MRT liefert nach stumpfem HWS-Trauma oft keine relevanten Zusatzinformationen}

\begin{abstract}
Die Computertomografie(CT)-Diagnostik gehört bei Patienten, die ein stumpfes Trauma der Halswirbelsäule erlitten haben, heutzutage zum Standard. Um die diagnostische Sicherheit zu erhöhen, wird vielfach die Magnetresonanztomografie (MRT) als weiteres bildgebendes Verfahren eingesetzt. Ihr Zusatznutzen ist einer aktuellen Metaanalyse zufolge allerdings minimal.
\end{abstract}

A jay Malhotra von der Yale School of Medicine in New Haven und Kollegen haben 23 Studien ausgewertet, in denen insgesamt 5.286 Patienten nach einem stumpfem Trauma der Halswirbelsäule (HWS) und negativem CT-Befund noch per MRT untersucht und nachbeobachtet wurden. In der MRT ergaben sich in 792 Fällen zusätzliche Befunde, was einer Rate von $15 \%$ entspricht. Eine gemäß der jeweiligen Studiendefinition „instabile Verletzung“, die in der CT unentdeckt geblieben waren, fand sich in der MRT bei 16 Patienten. Dies entspricht einer Inzidenz von $0,3 \%$.

Problematisch war, dass die Definitionen für Instabilität teilweise deutlich differierten: Einige Autoren beriefen sich auf die Klassifikation nach White und Panjabi; bei anderen galt eine Verletzung dann als instabil, wenn sie „mehr als eine Struktur der HWS“ betraf.
Eine Arbeit benutzte den Begriff „klinisch relevante Verletzung“, ohne diesen genauer $\mathrm{zu}$ definieren, eine weitere nahm den sogenannten Flexion Extension Film (FEF) als Grundlage.

Auch beim Timing gab es große Unterschiede: So wurde die MRT in manchen Studien innerhalb von 72 Stunden nach der CT durchgeführt, teilweise aber auch erst viel später. Das maximale Intervall betrug 39 Tage.

Um der erheblichen Heterogenität in den Studien Rechnung zu tragen, führten Malhotra und Kollegen eine zusätzliche Analyse durch, in der sie die Patienten zunächst in zwei Gruppen teilten: ansprechbare und solche mit gestörtem Bewusstsein. Für beide Gruppen sowie für jede Studie einzeln wurden dann Durchschnittswerte berechnet und diese nach dem Kehrwert der Varianz gewichtet. Aus diesen Berechnungen ergab

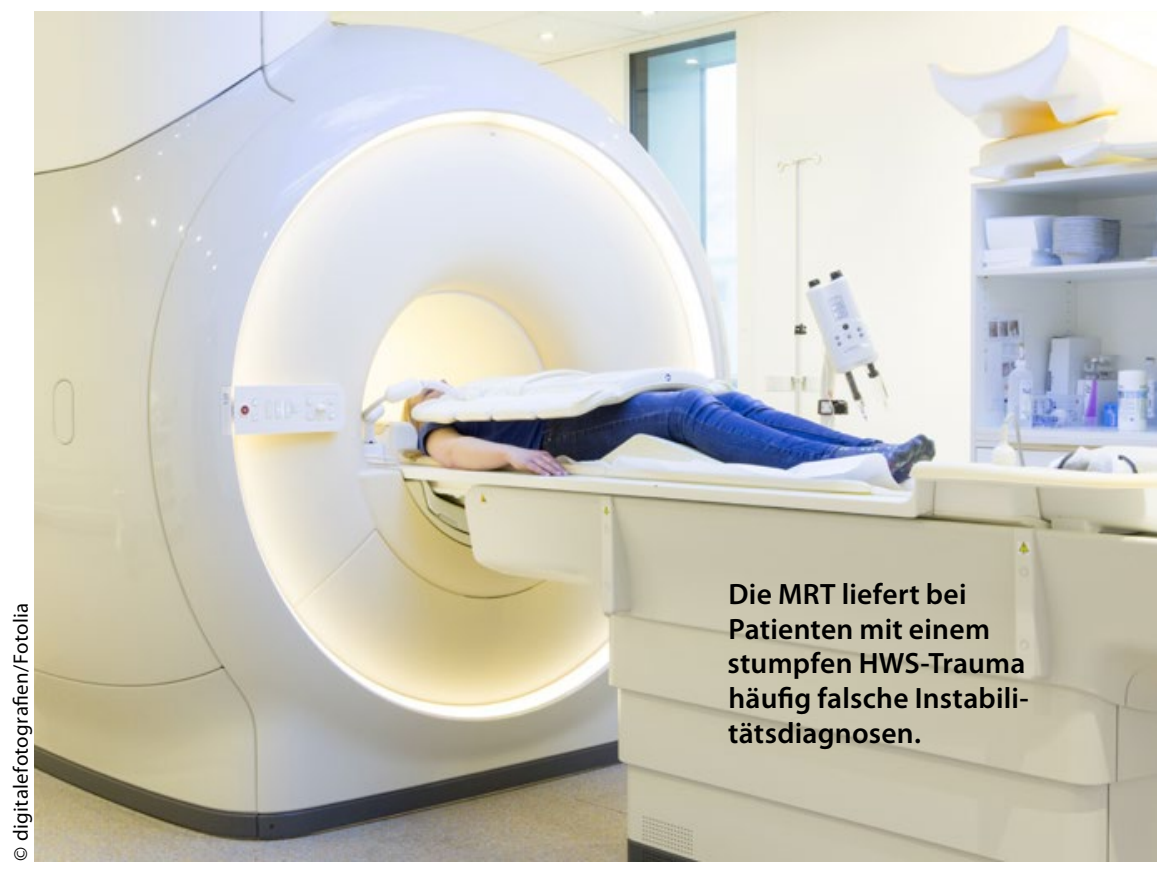

sich eine gepoolte positive Befundrate von $0,0029 \%$.

Für die insgesamt 3.370 bewusstseinsgestörten Patienten lag die Befundrate bei $0,12 \%$; für die 1.387 ansprechbaren waren es $0,72 \%$. Die gepoolten positiven Raten betrugen dagegen 0,017 beziehungsweise $0,011 \%$.

Für die Autoren ist das insofern nachvollziehbar, als Studien mit wachen $\mathrm{Pa}$ tienten, bei denen eine instabile Verletzung gefunden wurde, wesentlich geringere Teilnehmerzahlen hatten als solche, die nur nicht ansprechbare Teilnehmer berücksichtigten. Zum einen, so die Forscher, gehe man bei bewusstseinsgestör ten Patienten von einem massiveren Trauma aus, zum anderen sei eine MRT gemäß den aktuell gültigen Leitlinien der East Association for the Surgery of Trauma (EAST) nur bei Letzteren überhaupt indiziert. Nur in elf Fällen können sich die Autoren der Metaanalyse unter Berücksichtigung der Studiendetails der Einschätzung anschließen, dass es sich bei den in der MRT zusätzlich entdeckten HWS-Läsionen tatsächlich um eine instabile Verletzung handelte.

Fazit: „Die MRT war in den von uns analysierten Studien mit einer hohen Rate falsch positiver Befunde behaftet", resümieren Malhotra und sein Team. In weiterführenden Untersuchungen hätten sich viele der Befunde als nicht instabil herausgestellt. In den meisten Fällen habe es sich um ligamentöse Verletzungen gehandelt, diese seien jedoch oft nicht therapiebedürftig. Nicht zuletzt müsse man sich fragen, ob man zumindest in der Akutphase mit der MRT selbst nicht noch Schaden beim Patienten anrichte, von den Kosten ganz zu schweigen. Letztlich, so die Experten, hänge der Nutzen der MRT bei Patienten nach einem stumpfen HWS-Trauma von einer Vielzahl von Variablen ab, von denen man die meisten zum gegenwärtigen Zeitpunkt noch nicht näher bestimmen könne. Weitere, vor allem prospektive Studien zum Thema seien demzufolge nötig.

Elke Oberhofer

Malhotra, A., Wu, X., Kalra, V.B. et al. Eur Radiol (2016). doi:10.1007/s00330-016-4426-Z 